\title{
Retrograde CTO PCI
}

\author{
Vijay Bang* \\ Interventional Cardiologist, Lilavati Hospital, India
}

Submission: February 07, 20176; Published: February 22, 2017

*Corresponding author: Vijay Bang, Interventional Cardiologist, Lilavati Hospital, Global Hospital and Fortis Hospital, Mumbai, India, Email: shivvhb@yahoo.com

\section{Introduction}

The art and technique of CTO PCI have been growing at an amazing pace since past one decade. Despite many CTO specific hardware and techniques like CTO specific wires, parallel wire technique, IVUS guided side branch access, sub-intimal tracking and re-entry technique (STAR), micro-channel technique and limited antegrade sub-adventitial tracking (LAST) have been proven to be useful in antegrade approach; about $20-30 \%$ of CTOs require retrograde approach, as advocated first by Hartzler (1990) and later championed by Japanese operators (2006-2014). Retrograde approach through collateral channels (CC) is gaining acceptance to improve the success rates of CTO recanalization from $65-70 \%$ to $90-94 \%$ as distal cap is softer because it is exposed to low haemodynamic pressure shear stress from collateral flow whereas proximal cap is harder as it is exposed to high aortic pressure shear stress. Retrograde approach until recently was suggested only after antegrade approach failed but now direct retrograde approach is slowly gaining momentum in a setting of unfavorable CTO anatomy e.g. blunt or ambiguous proximal cap, long occlusion, severe calcification (CTA), tortuosity and side branches at the cap.

Retrograde CTO PCI Procedure Involves Systematic Stepwise Approach to Demystify the Art Of CTO PCI

\section{CC analysis}

It is vital to do careful analysis of CC on coronary angiography performed by mandatory simultaneous dye injections in donor and recipient artery using either bi-femoral or one femoral/one radial access. Septal CC is preferred to epicardial CC as it is less tortuous and very less fatal if it perforates [1].

\section{Retrograde CC crossing}

Short retrograde guide $(90 \mathrm{~cm})$ is preferred so that equipment length in retrograde approach does not fall short in reaching CTO artery. Selected CC is wired proximally using regular workhorse wire to pass through micro-catheter (Corsair or Fine-cross). Corsair $(150 \mathrm{~cm})$ is preferred as it is hydrophilic, serves as CC dilator and provides exceptional CC tracking with gentle clockwise and anticlockwise movements. Once the Corsair is positioned in proximal end of CC, workhorse wire is exchanged to Fielder XT or FC or Pilot 50 wire. Tapered tip wire like XT can track through tiny CC as well. Distal $1 \mathrm{~mm}$ tip of wire is generally shaped to 30-45 degree angle to smoothly surf through CC. First wire is navigated through CC and then Corsair is carefully and gently tracked over wire into distal true lumen of the CTO. Generally small perforation with wire alone is benign in septal CC [2].

\section{Crossing of CTO}

Once the wire and Corsair reaches close to distal cap of CTO, crossing of CTO is attempted with the same wire. If the distal cap is firm or hard by tactile sensation then stiffer wire like Confianzo Pro 12 may be exchanged and attempted to penetrate distal cap without wasting too much time on soft wire attempt, as time and contrast efficiency is extremely vital during retrograde CTO PCI to prevent CIN, acute LVF and high radiation exposer. If stiffer wire fails to penetrate then it is advisable to exchange it again with Fielder FC or XT to proceed with knuckle wire technique which entails creating a small loop at the distal end of wire by just pushing the wire without rotation to dissect the sub-intimal or sub-adventitial space. It is advisable to avoid wire rotation for creating a loop as it can lead to knotting of wire which then is entrapped in sub-intimal space and impossible to remove, so due care should be taken not to rotate the wire during loop formation. Then CART or reverse CART is performed which entails balloon inflation in sub-intimal or sub-adventitial space. The reverse CART is more successful and preferable in majority of cases as balloon is inflated over the antegrade wire hence there is no limitation on size of balloon diameter to be inflated in subintimal or sub-adventitial space for successful re-entry in true lumen. Whereas in CART, balloon is inflated over the retrograde wire hence balloon size is limited to 1.25 or $1.5 \mathrm{~mm}$ as it has to 
pass through CC. The inflation of the proper size balloon in subintimal or sub-adventitial space is key to success for re-entry in true lumen. Diameter of balloon can be assessed on IVUS images. Hybrid approach to CTO focuses on opening the occluded vessel using all feasible techniques antegrade, retrograde, true to true lumen crossing or re-entry in most safe, effective \& efficient way. Latest is Stingray (Boston Scientific) balloon which is flat on one surface hence if inflated in sub-intimal or sub-adventitial space, it inflates only on softer side (intima) and remains flat on harder side (adventitia) as biologically intima is softer and adventitia is harder. Tornus or Crossboss is used only if stiffer antegrade wire fails to penetrate proximal cap despite micro-catheter or OTW balloon support. US FDA and CE approved Crossboss and Stingary balloon is currently not available in India. Two new microcatheters became available this year namely

a) Turnpike (vascular solutions).

b) MicroCross (Roxwood Medical).

Turnpike has dual layer bidirectional coil which facilitates torque transmission \& prevents kinking. Moreover two support catheters are now available namely

a. MultiCross (Rexwood Medical).

b. CenterCross (Rexwood Medical).

Both support catheters have a stabilizing self-expanding scaffold that is deployed proximal to target lesion for providing anchor support to manipulate CTO penetration with harder wire. MultiCross contains three microcatheters within the scaffold. CenterCross has single large central lumen that can accommodate a microcatheter. Both catheters require an adequate landing zone in target vessel.

\section{Externalization or Snaring retrograde wire and performing angioplasty}

If reverse CART technique is successful then angioplasty is performed on antegrade wire and retrograde equipment is removed. Whereas if direct retrograde wire technique or CART is successful to lead in to true lumen then retrograde wire needs to be externalized by carefully advancing it along with Corsair through CTO till it reaches antegrade guide, at this stage retrograde wire is exchanged to Fielder RG $(300 \mathrm{~cm})$ double length wire which is slowly advanced in to enter antegrade guiding and then externalized once it reaches the haemostatic cap of antegrade guiding and followed by angioplasty over RG wire through antegrade guide. If the retrograde wire fails to enter antegrade guiding then it may pop out into ascending aorta which may require snaring of retrograde wire using either a three loop En Snare (18-30mm) or Memory snare and then externalized. Angioplasty is then performed routinely through antegrade guide catheter. Important here is not to withdraw Corsair from the CC till the angioplasty is over as undue stress on retrograde wire during passage of balloon or stent may damage CC. Once the angioplasty is over, Corsair can be safely removed and angiographic pictures are taken with simultaneous injection through both guides to see the final angioplasty result and to evaluate any damage to CC channel or both coronary ostia due to prolonged complex procedure. Little contrast staining of septum is not infrequent and does not cause any haemodynamic compromise. If there is damage to any of the coronary ostia due to guide movements, it should be treated in appropriate manner. The collateral channels tend to close immediately after antegrade flow in CTO artery is established hence it should not be mistaken for CC damage or thrombosis. Due care should be taken to maintain ACT 300+ throughout the procedure to prevent thrombus formation in retrograde equipment or concerned vessels. Unfractionated heparin is preferred during retrograde approach as it has an antidote to reverse anticoagulation action in case of CC perforation [3].

\section{Conclusion}

Retrograde CTO PCI represents final frontier in interventional cardiology. The success in retrograde CTO PCI is due to the cutting edge advancement in hardware, technology and skills. Retrograde CTO PCI is now performed with high degree of safety, efficiency and success rates in experienced hands.

\section{References}

1. Tsuchkane E, Katoh O, Kimura M, Nasu K, Kinoshita Y, et al. (2010) The first clinical experience with a novel catheter for collateral channel tracking in retrograde approach for chronic coronary total occlusions. JACC Cardiovasc Interv 3(2): 165-171.

2. Wu EB, Chan WW, Yu CM (2008) Retrograde chronic total occlusion intervention: tips \& tricks. Catheter Cardiovasc Interv 72(6): 806-814.

3. Rathore S, Katoh O, Tsuchikane E, Oida A, Suzuki T, et al. (2010) A novel modification of the retrograde approach for the recanalization of chronic total occlusion of the coronary arteries intravascular ultrasound-guided reverse controlled antegrade \& retrograde tracking. JACC Cardiovasc Interv 3(2): 155-164. 
This work is licensed under Creative Commons Attribution 4.0 License DOI: 10.19080/JOCCT.2017.03.555609

\section{Your next submission with Juniper Publishers} will reach you the below assets

- Quality Editorial service

- Swift Peer Review

- Reprints availability

- E-prints Service

- Manuscript Podcast for convenient understanding

- Global attainment for your research

- Manuscript accessibility in different formats

( Pdf, E-pub, Full Text, Audio)

- Unceasing customer service

Track the below URL for one-step submission https://juniperpublishers.com/online-submission.php 